\title{
SHORT COMMUNICATION \\ INTERNATIONAL EVIDENCE FOR THE EFFECTIVENESS OF THE FRONT-OF-PACKAGE NUTRITION LABEL CALLED NUTRI-SCORE
}

\author{
Valentina A. Andreeva1, Manon Egnell'1, Mathilde Touvier ${ }^{1}$, Pilar Galan¹, Chantal Julia ${ }^{1,2}$, Serge Hercberg ${ }^{1,2}$ \\ ${ }^{1}$ Nutritional Epidemiology Research Group, Sorbonne Paris Nord University, Bobigny, France \\ ${ }^{2}$ Department of Public Health, Avicenne Hospital, Bobigny, France
}

\begin{abstract}
SUMMARY
Objectives: Front-of-package nutrition labels are intended to easily convey to consumers comprehensible information about the nutritional composition of pre-packaged food and are thus a tool in the combat against the growing prevalence of nutrition-related disorders, such as obesity, type 2 diabetes, cardiovascular disease, and some types of cancer. The objective of the present narrative review was first to describe Nutri-Score and then to synthesize some of the international scientific evidence for its effectiveness. Guided by scientific data and collective expertise, France formally adopted labelling of pre-packaged food with the 5-colour Nutri-Score label in October 2017 and that move was later followed by Belgium, Spain, Germany, Switzerland, the Netherlands, and Luxembourg.

Methods: This article synthesizes evidence from several countries regarding the effectiveness of Nutri-Score and the associated individual-level diet quality index in terms of attitude/behaviour- and health-related outcomes. It also addresses criticism levied at the label.

Results: The effectiveness of Nutri-Score has been demonstrated in terms of consumer ability to correctly classify food according to its nutritional quality, the nutritional quality of actual and intended food purchases, and portion size choices. In addition, consumption of foods that are less favourably rated on the Nutri-Score scale has been prospectively associated with chronic disease risk (cancer, cardiovascular disease, metabolic syndrome, etc.).

Conclusion: The adoption and implementation of a uniform front-of-package label such as the scientifically validated Nutri-Score on pre-packaged foods/beverages across Europe could be beneficial to consumers at the point of purchase and could help reduce the incidence of diet-related chronic diseases by means of improvement in diet quality.
\end{abstract}

Key words: Nutri-Score, nutritional quality, front-of-package labels, chronic disease, nutrition policy, Europe

Address for correspondence: V. A. Andreeva, Nutritional Epidemiology Research Group (EREN), UMR U1153 Inserm/U1125 Inrae/Cnam, Sorbonne Paris Nord University, 74 Marcel Cachin, 93017 Bobigny, France. E-mail: v.andreeva@eren.smbh.univ-paris13.fr

https://doi.org/10.21101/cejph.a6239

\section{INTRODUCTION}

In effort to counter the detrimental impact of the highly prevalent energy-dense/nutrient-poor diets, the World Health Organization (WHO) has provided not only individual but also industry-level guidelines that include ensuring the availability and affordability of nutritious food worldwide and reformulating food products for the purpose of reducing fat/sugar/sodium content (1). The nutritional composition of pre-packaged food can be easily conveyed to the consumer by means of front-of-package labels (FOPL). A wide range of FOPL systems has been developed and implemented on a voluntary or mandatory basis worldwide (2). FOPL either provide only numerical data about the content/ quantity of one or several nutrients or display a graphical and/or colour-coded synthesis of the food's overall nutritional composition and quality (3). The Nutri-Score FOPL (also known as the 5 -colour $\log$ ) falls into the latter category. The objective of the present narrative review was first to describe Nutri-Score and then to synthesize some of the international scientific evidence for its effectiveness.

\section{Description of Nutri-Score}

Nutri-Score was put forward by the French public health agency, based on scientific evidence as well as expertise of the High Council for Public Health (4). It is a simple, coloured FOPL that synthesizes the numerical information from the mandatory nutrition declaration available on the back of each package. It features five categories of nutritional quality (Fig. 1), pairing the letters from "A" through " $E$ " with colours ranging from dark green (associated with higher nutritional quality) to red (associated with lower nutritional quality). The categories are set off by means of a validated algorithm developed in 2005 by a research team at Oxford University for the UK Food Standards Agency (FSA) (5). Adaptation of the algorithm (regarding beverages, added lipids and cheese) and the cut-off values for 


\section{NUTRI-SCORE \\ (A) B C D} NUTRI-SCORE A B CDE A B B C D E

Fig. 1. Five nutritional quality categories of Nutri-Score.

each category were provided by the French High Council for Public Health (6).

The nutrient profiling system (NPS) underlying the Nutri-Score assigns points according to the nutrient content in $100 \mathrm{~g}$ of food or $100 \mathrm{ml}$ of beverage. Dietary components considered "favourable" for health are fruit, vegetables, legumes, nuts, fiber, vegetable fats and protein; depending on their respective quantity, each is given a score between -5 and 0 points. In turn, dietary components considered "unfavourable" for health are sugars, saturated fat, sodium, and total energy. Depending on the respective quantity, from 0 to 10 points are attributed to each component. The total score is obtained by subtracting the absolute value of the "favourable" points from the "unfavourable" points (theoretical range: -15 to +40 ). A lower number of points corresponds to a higher nutritional quality. Overall, Nutri-Score is viewed as extending the trafficlight system and as meeting the US Institute of Medicine (now the National Academy of Medicine) requirements for successful food classification, as its comprehension does not require any specific nutrition knowledge given the simple ranking symbols that are identifiable, easy to remember, and serve as guidance for product comparison (7).

This food-level FOPL system and the nutrient profile that it conveys were subsequently used as the basis for an individual level diet quality index (FSA-NPS) obtained by first estimating the average nutrient profile at the food level and then aggregating those data at the individual level. This individual-level dietary index has the same theoretical range as Nutri-Score, with lower scores corresponding to better overall diet quality (8).

Nutri-Score, which has been acknowledged by the WHO (9), has been the official nutritional quality information system in France since October 2017, even though its implementation is voluntary given the European regulations currently in place. Yet, on the European level, Belgium (August 2017), Spain (November 2018), Germany (September 2019), Switzerland (September 2019), the Netherlands (November 2019), and Luxembourg (February 2020) have also formally adopted Nutri-Score.

\section{Effectiveness Regarding Nutrition Attitude/Behav- iour Outcomes}

A recent 3-arm trial (Nutri-Score, Multiple Traffic Lights and no-FOPL control) among 154 online grocery shoppers in Singapore revealed the superior performance of Nutri-Score when the objective was diet quality improvement, measured with a modified Alternative Healthy Eating Index 2010 (10). Next, a French trial with 691 participants tested five FOPL (Multiple Traffic
Lights, Health Star Rating, Reference Intakes/Guideline Daily Amount, Nutri-Score, and Simplified Food Labelling System) in a controlled experimental food store and likewise showed the advantages of Nutri-Score regarding the nutritional quality of the items in the shopping cart (11). In addition, the performance of Multiple Traffic Lights, Reference Intakes/Guideline Daily Amount, Nutri-Score, and Simplified Food Labelling System was evaluated over a 10-week period in 2016 in 60 actual supermarkets (20 of which were set as "controls") across 4 French regions ( $n=1,748,503$ sales receipts) (12). Overall, the results showed that FOPL have a measurable impact on food purchasing behaviours. Specifically, Nutri-Score, Multiple Traffic Lights and Simplified Food Labelling System were respectively associated with a $4.5 \%, 3.9 \%$, and $3.3 \%$ increase in the nutritional quality of the purchased food/beverage items (12). The advantageous performance of Nutri-Score was especially marked with respect to the purchasing behaviours of consumers who tended to choose the most inexpensive products (12).

The potential influence of Nutri-Score on food-purchasing decisions and the nutritional quality of the actual purchases was examined by means of a randomized field intervention in a university cafeteria in Bogotá, Colombia (7). The investigators randomly provided information about the Nutri-Score of the products available. The trial included 257 participants in the active group and 228 controls. The results showed that the active group spent, on average, $21 \%$ or $\$ 0.26$ more on healthier items and had chosen products with a higher protein content compared to controls. Further, information about Nutri-Score at the time of purchase had increased the cafeteria's sales, which the authors highlighted as evidence for a financial incentive that could potentially facilitate the implementation of Nutri-Score (7).

The choice of portion size according to one of four FOPL conditions (Nutri-Score, Multiple Traffic Lights, Evolved Nutrition Label, and no-FOPL condition) was examined in an online experiment conducted among 25,772 participants in the French NutriNet-Santé cohort (13). The study featured images of food products belonging to three categories (sweet biscuits, cheese and sweet spreads), presented in four different portion sizes. Participants were asked to select an image corresponding to the portion size that they would choose and also to indicate the number of portions desired. Overall, compared to the no-FOPL situation, Nutri-Score, followed by Multiple Traffic Lights, significantly and consistently lowered the portion sizes selected (13).

Finally, consumers' ability to correctly classify food according to its nutritional quality was investigated in an international experimental study featuring five FOPL (French Nutri-Score system, British Multiple Traffic Lights system, Australian Health Star Rating system, industry-advocated Reference Intakes system, and Chilean Warning system) and three different food categories (breakfast cereal, pizza and cake) (14). That study involved 12,000 consumers from 12 countries: Argentina, Australia, Bulgaria, Canada, Denmark, France, Germany, Mexico, Singapore, Spain, the United Kingdom, and the United States. The results showed that all five FOPL improved participants' ability to correctly classify products according to their respective nutritional quality. Across the twelve countries, and especially in the European countries, Nutri-Score emerged as the most effective system, followed by Multiple Traffic Lights, Health Star Rating, Warning and finally the Reference Intakes (14). 


\section{Effectiveness Regarding Health-related Outcomes}

A number of morbidity and mortality outcomes have been studied with respect to their association with the Nutri-Score-based individual-level dietary index (FSA-NPS). For example, cancer risk was studied among 471,495 adults from 10 European countries (Denmark, France, Germany, Greece, Italy, the Netherlands, Norway, Spain, Sweden, and the United Kingdom) involved in the European Prospective Investigation into Cancer and Nutrition cohort (EPIC, 1992-2014, median follow-up 15.3 years) (15). The results revealed that a lower dietary index (reflecting consumption of food/beverages ranked less favourably with Nutri-Score) was associated with a higher risk of cancer overall, and particularly cancer of the colon-rectum, upper aero-digestive tract, stomach, lung (among men), liver, and postmenopausal breast cancer among women (15). Cancer risk and several other health outcomes were investigated in the French SU.VI.MAX cohort ( $\mathrm{N}=6,850$, 13 years of follow-up). Overall, the results showed that a lower dietary index was significantly associated with a higher risk of cancer overall (16), cardiovascular disease (especially in at-risk individuals such as those who are smokers and/or physically inactive) (17), metabolic syndrome (18), and weight gain (19). Further, a higher dietary index was cross-sectionally associated with a somewhat lower risk of oral health problems in a sample from the French NutriNet-Santé cohort (20).

Finally, a study applying the preventable risk integrated macrosimulation model estimated the impact of modification in dietary intake resulting from FOPL exposure (Nutri-Score, Health Star Rating, Multiple Traffic Lights, Reference Intakes, Simplified Food Labelling System) on mortality from diet-related chronic diseases (21). Overall, it was estimated that $3.4 \%$ of mortality was avoidable through use of Nutri-Score, compared with $2.8 \%$ for Health Star Rating, 1.9\% for Reference Intakes, $1.6 \%$ for Multiple Traffic Lights, and 1.1\% for Simplified Food Labelling System (21).

\section{Discussion of Criticism Levied at Nutri-Score}

Critics of Nutri-Score have pointed out several issues, such as the absence of vitamin/mineral and sustainability/environmental impact measures from the scoring algorithm (in fact, the score occasionally categorizes more favourably certain more processed than less processed food), the absence of information on additives or pesticides in the food, the score being calculated per $100 \mathrm{~g} / \mathrm{ml}$ and not per portion, etc. Some of these issues have already been addressed $(22,23)$. Below we address the issue of additives and pesticides which thus far has been one of the most important criticisms.

At present, no food labelling system has the capacity to include information on additives or pesticides because the current level of scientific knowledge does not permit the development of an indicator that can reflect all food composition aspects in a FOPL. In Europe in particular, over 330 food additives - in the form of artificial sweeteners, emulsifiers, dyes, stabilizers, preservatives, anti-microbial agents, antioxidants, etc. - have been authorized for use in conventional food/beverage products (versus 48 additives authorized for use in organic products) (24). It has been estimated that over half of the food/beverage products available on the French market contain a minimum of one additive with the product categories most likely to contain additives being artificially sweetened beverages, ice cream, industrially made sandwiches, cakes, and biscuits (i.e., ultra-processed foods, which may also contain neo-formed compounds and substances migrating from the packaging). Thus far, evidence for the adverse effects of individual additives (e.g., nitrates/nitrites, glutamate, bixin, titanium dioxide, etc.) on metabolic, endocrine and other health outcomes has come from animal and/or cell culture research; moreover, few studies have explored the potential interaction among different additives and the food matrix overall (24).

In turn, human exposure to pesticides is ubiquitous and may occur via inhalation, ingestion, dermal contact or across the placenta (25). Similarly to additives, pesticides constitute a very large group of chemical substances with disparate degrees of toxicity (26). Information about the potential health effects of pesticides is largely provided by toxicological research using animal or in vitro studies performed in strictly controlled conditions (e.g., diet, air quality) (25). In addition, the animals in those studies are typically exposed to one agent at a time, with additive/synergistic/ potentiating effects of multiple agents being rarely explored. The sparse data on the effect of pesticides in humans come from occupational health research (25).

Despite the present lack of solid evidence for the long-term chronic disease risk associated with the intake of additives/pesticides, current public health nutrition policies can nonetheless advocate for the consumption of home-cooked, unprocessed and/or organic food, foods/beverages with the most favourable Nutri-Score, and the use of an accompanying list of additives and/or pesticides. Meanwhile, studies are presently underway to evaluate the direct and indirect links between intake of additives/ pesticides (alone or in combination) and chronic disease risk. In the near future, it might be possible to compile convincing evidence regarding specific substances and/or mixtures that portend health risk. Such knowledge could then be integrated in nutrition policies and in an updated version of Nutri-Score.

\section{CONCLUSION}

A 2018 World Health Organization report highlighted the fact that FOPL that are simple, noticeable and understandable have the potential to both urge consumers to make informed healthier food choices and drive product reformulation by manufacturers (27). Hence, advocating for the application of Nutri-Score on the European food/beverage market is grounded in the extensive validation of its nutrient profiling capacity and its graphical format; moreover, international evidence is accumulating regarding its effectiveness in terms of attitude, behaviour, and health-related outcomes.

\section{Conflict of Interests}

None declared

\section{REFERENCES}

1. World Health Organization. Obesity and overweight [Internet]. Geneva: WHO; 2020 [cited 2021 Feb 13]. Available from: https://www.who.int/ news-room/fact-sheets/detail/obesity-and-overweight.

2. World Cancer Research Fund International. Lessons on implementing a robust front-of-pack food label [Internet]. London: WCRF International 
[cited 2021 Feb 13]. Available from: https://www.wcrf.org/int/policy/ our-publications/lessons-implementing-front-of-pack-label.

3. Kanter R, Vanderlee L, Vandevijvere S. Front-of-package nutrition labelling policy: global progress and future directions. Public Health Nutr. 2018;21(8):1399-408

4. Santé Publique France. Nutri-Score [Internet]. Paris: Santé Publique France; 2020 [cited 2021 Feb 13]. Available from: https:/www.santepubliquefrance.fr/en/nutri-score.

5. Rayner M, Scarborough P, Boxer A, Stockley L. Nutrient profiles: development of final model. London: Food Standards Agency; 2005.

6. Haut Conseil de la Santé Publique. Information on the nutritional quality of food products [Internet]. Paris: HCSP; 2015 [cited 2021 Feb 13]. Available from: http://www.hcsp.fr/Explore.cgi/avisrapportsdomaine?clefr=519. (In French.)

7. Mora-García CA, Tobar LF, Young JC. The effect of randomly providing Nutri-Score information on actual purchases in Colombia. Nutrients. 2019:11(3):491. doi: 10.3390/nu11030491.

8. Julia C, Touvier M, Méjean C, Ducrot P, Péneau S, Hercberg S, et al. Development and validation of an individual dietary index based on the British Food Standard Agency Nutrient Profiling System in a French context. J Nutr. 2014;144(12):2009-17.

9. World Health Organization. France becomes one of the first countries in Region to recommend colour-coded front-of-pack nutrition labelling system [Internet]. Copenhagen: WHO Regional Office for Europe; 2017 [cited $2021 \mathrm{Feb}$ 13]. Available from: http://www.euro.who.int/en/ countries/france/news/news/2017/03/france-becomes-one-of-the-firstcountries-in-region-to-recommend-colour-coded-front-of-pack-nutritionlabelling-system.

10. Finkelstein EA, Ang FJL, Doble B, Wong WHM, van Dam RM. A randomized controlled trial evaluating the relative effectiveness of the Multiple Traffic Light and Nutri-Score front of package nutrition labels. Nutrients. 2019;11(9):2236. doi: 10.3390/nu11092236.

11. Crosetto P, Lacroix A, Muller L, Ruffieux B. Modifications of food purchases in response to five nutrition simplified labelling. Cah Nut Diet. 2017;52(3):129-33. (In French.)

12. French Ministry of Social Affairs and Health. Simplified nutrition labelling: implementation of the law on modernising our health system (article 14-II). Report of the steering committee for assessment under actual buying conditions. Paris: French Ministry of Social Affairs and Health; 2017.

13. Egnell M, Kesse-Guyot E, Galan P, Touvier M, Rayner M, Jewell J, et al. Impact of front-of-pack nutrition labels on portion size selection: an experimental study in a French cohort. Nutrients. 2018;10(9):1268. doi: 10.3390/nu10091268.

14. Egnell M, Talati Z, Hercberg S, Pettigrew S, Julia C. Objective understanding of front-of-package nutrition labels: an international comparative experimental study across 12 countries. Nutrients. 2018;10(10):1542. doi: 10.3390/nu10101542.

15. Deschasaux M, Huybrechts I, Murphy N, Julia C, Hercberg S, Srour B, et al. Nutritional quality of food as represented by the FSAm-NPS nutrient profiling system underlying the Nutri-Score label and cancer risk in Europe: results from the EPIC prospective cohort study. PLoS Med. 2018;15(9):e1002651. doi: 10.1371/journal.pmed.1002651.

16. Donnenfeld M, Julia C, Kesse-Guyot E, Méjean C, Ducrot P, Péneau S, et al. Prospective association between cancer risk and an individual dietary index based on the British Food Standards Agency Nutrient Profiling System. Br J Nutr. 2015;114(10):1702-10.

17. Adriouch S, Julia C, Kesse-Guyot E, Méjean C, Ducrot P, Péneau S, et al. Prospective association between a dietary quality index based on a nutrient profiling system and cardiovascular disease risk. Eur J Prev Cardiol. 2016;23(15):1669-76.

18. Julia C, Fezeu LK, Ducrot P, Méjean C, Péneau S, Touvier M, et al. The nutrient profile of foods consumed using the British Food Standards Agency Nutrient Profiling System is associated with metabolic syndrome in the SU.VI.MAX cohort. J Nutr. 2015;145(10):2355-61.

19. Julia C, Ducrot P, Lassale C, Fezeu L, Méjean C, Péneau S, et al. Prospective associations between a dietary index based on the British Food Standard Agency nutrient profiling system and 13-year weight gain in the SU.VI.MAX cohort. Prev Med. 2015;81:189-94.

20. Andreeva VA, Egnell M, Galan P, Feron G, Hercberg S, Julia C. Association of the dietary index underpinning the Nutri-Score label with oral health: preliminary evidence from a large, population-based sample. Nutrients. 2019;11(9):1998. doi: 10.3390/nu11091998.

21. Egnell M, Crosetto P, d'Almeida T, Kesse-Guyot E, Touvier M, Ruffieux $\mathrm{B}$, et al. Modelling the impact of different front-of-package nutrition labels on mortality from non-communicable chronic disease. Int J Behav Nutr Phys Activity. 2019;16(1):56. doi: 10.1186/s12966-019-0817-2.

22. Julia C, Charpak Y, Rusch E, Lecomte F, Lombrail P, Hercberg S. Promoting public health in nutrition: Nutri-Score and the tug of war between public health and the food industry. Eur J Public Health. 2018;28(3):396-7.

23. Julia C, Etilé F, Hercberg S. Front-of-pack Nutri-Score labelling in France: an evidence-based policy. Lancet Public Health. 2018;3(4):e164. doi: 10.1016/S2468-2667(18)30009-4.

24. Chazelas E, Deschasaux M, Srour B, Kesse-Guyot E, Julia C, Alles B, et al. Food additives: distribution and co-occurrence in 126,000 food products of the French market. Sci Rep. 2020;10:3980. doi: 10.1038/ s41598-020-60948-w.

25. Gilden RC, Huffling K, Sattler B. Pesticides and health risks. J Obstet Gynecol Neonatal Nurs. 2010;39(1):103-10.

26. Bretveld RW, Thomas CMG, Scheepers PTJ, Zielhuis GA, Roeleveld N. Pesticide exposure: the hormonal function of the female reproductive system disrupted? Reprod Biol Endocrinol. 2006;4:30. doi: 10.1186/14777827-4-30.

27. Kelly B, Jewell J. What is the evidence on the policy specifications, development processes and effectiveness of existing front-of-pack food labelling policies in the WHO European Region? Health Evidence Network synthesis report 61. Copenhagen: WHO Regional Office for Europe; 2018. 